\title{
Two-coordinate pointing and tracking system for an infrared Fourier- transform spectrometer
}

\begin{abstract}
Alexey Shakun, Andrey Kungurov, Oleg Sazonov, Igor Stupin, Gabriele Arnold, et al.
\end{abstract}

Alexey Shakun, Andrey Kungurov, Oleg Sazonov, Igor Stupin, Gabriele Arnold, Alexey Grigoriev, Oleg Korablev, "Two-coordinate pointing and tracking system for an infrared Fourier-transform spectrometer," Proc. SPIE 11128, Infrared Remote Sensing and Instrumentation XXVII, 111280H (9 September 2019); doi: 10.1117/12.2535444

SPIE Event: SPIE Optical Engineering + Applications, 2019, San Diego, California, United States 


\title{
Two-coordinate pointing and tracking system for an infrared Fourier- transform spectrometer
}

\author{
Alexey Shakun*a , Andrey Kungurov ${ }^{\mathrm{a}}$, Oleg Sazonov ${ }^{\mathrm{a}}$, Igor Stupin ${ }^{\mathrm{a}}$, Gabriele Arnold ${ }^{\mathrm{b}}$, Alexey \\ Grigoriev $^{\mathrm{a}}$, Oleg Korablev ${ }^{\mathrm{a}}$ \\ ${ }^{a}$ Space Research Institute (IKI), 84/32 Profsoyuznaya, 117997 Moscow, Russia; \\ ${ }^{\mathrm{b}}$ Deutsches Zentrum für Luft- und Raumfahrt e.V. (DLR), Institute of Planetary Research, \\ Rutherfordstrasse 2, 12489 Berlin, Germany. \\ *avshakun@iki.rssi.ru; phone+74953336245
}

\begin{abstract}
We describe a 1-inch aperture pointing system realized with a rope transmission driven by geared stepper motors for operation at the surface of Mars. The system is intended to serve in the Fourier-transform spectrometer to observe both the sun through the atmosphere and the thermal emission of the atmosphere. The scanner covers a full $2 \pi$ field of view (the upper hemisphere). The accuracy of $0.1 \mathrm{mrad}(\sim 21 \mathrm{arcsec})$ for the sun tracking is achieved using an automatic control system with an IR array sensor $(32 \times 32)$ in the loop. The optics of the pointing system consists of three flat mirrors and a germanium inlet window. The mass of the subsystem does not exceed $0.8 \mathrm{~kg}$. The design accounts for the necessity of dust protection in the Martian environment. The effect of a finite accuracy of the pointing and tracking on the instrument signal to noise ratio is modeled.
\end{abstract}

Keywords: Fourier-transform spectrometer, pointing system, Sun tracking system, IR remote sensing

\section{INTRODUCTION}

A scanner/positioner is a subsystem usually accompanying a spaceborne infrared (IR) Fourier-transform spectrometer (FTIR). Occasional pointing of the instrument's instantaneous field of view (IFOV) to the calibration source or to open space is required for the calibration purpose. If an instrument aims at the sun disk, a bright IR source allowing to obtain a high spectral resolution, it has to be equipped with a precise two coordinate pointing system. Requirements for such system are high precision, low mass, and capability to withstand the launch and landing mechanical loads. The development and prototyping of the two coordinates pointing system is a part of activity supporting the advancement of the FTIR FAST (Fourier for Atmospheric Species and Temperature) [2, 3], the instrument of the science payload to fly at the ExoMars landing platform [4]. The main science goal of FAST is to measure trace gases in the Martian atmosphere utilizing Sun observation with high spectral resolution. Presence of minor organic species in the Martian atmosphere can result from the geological or biological activity on Mars [5]. Also, FAST aims on long term monitoring of climatological parameters (aerosol and temperature profiles) [6,7]. Sun observation with lower spectral resolution focus at aerosol (dust and water ice) studies, and Mars atmosphere observation $[8,9]$ with no Sun in the instrument IFOV, provide thermal sounding, the most precise within the planetary boundary layer.

The 1-inch interferometer of FAST [2,3] is based on a Michelson scheme with retroreflectors instead of the flat mirrors. To ensure the required (150-mm) maximal optical path difference (MOPD) a single-axis robot with a geared stepper motor is applied. One measurement of a partially symmetric interferogram in the trace gases regime lasts $\sim 500 \mathrm{~s}$. The angular displacement of the Sun as observed from the surface of Mars is significant and amounts to 23 arcsec. Therefore one of the requirements for the pointing system is the possibility of the Sun tracking with high accuracy. The final limitations on the tracking accuracy and the estimation of its effect on the instrument signal-to-noise ratio (SNR) are based on modeling. Another important problem, which has to be solved prior the Sun tracking, is to find the Sun in the upper hemisphere. Sun searching and tracking rely on the information from the IR array sensor, which is included

Infrared Remote Sensing and Instrumentation XXVII, edited by Marija Strojnik, Gabriele E. Arnold, Proc. of SPIE Vol. 11128, 111280H · @ 2019 SPIE · CCC code: 0277-786X/19/\$18 · doi: 10.1117/12.2535444 
into the operation loop. A simpler task for the pointing system is to point the instrument's IFOV to a specified direction within the upper hemisphere or to the calibration source.

To minimize the mass of the two-coordinate pointing and tracking system, we implement a rope drive. Different types of motors were considered and tested to get an optimal configuration of the mechanism with regard to the accuracy, mass, volume, power supply requirements, the complexity of the driving electronics. Testing also aimed to find out if a mechanical locker system is necessary to withstand launch and landing mechanical loads. A configuration with no locker required was found, but a version with the locker offered a more compact solution.

The present paper provides a detailed description of the high precision two-coordinate pointing and sun-tracking system for an FTIR developed for Martian landing platform [3]. The main technical solutions, tests, and modeling results are presented. A description of the pointing system operation algorithms is also given.

\section{POINTING SYSTEM GENERAL DESIGN}

The optical head of the two-coordinate pointing system includes two scanning and one fixed flat mirror (Fig. 1). Two geared stepper motors support rotation of the scanning mirrors via pulleys and tungsten wires (diameter of $0.4 \mathrm{~mm}$ ). Motor shafts are rigidly connected with the main pulleys. The wires are tightened so that rotation of more than $360^{\circ}$ is possible for each of the main pulleys. This rotation range allows covering the full upper hemisphere and the nadir direction. Software controls the rotation of each shaft within $360^{\circ}$ by means of encoders connected with the main pulleys to avoid the redundancy. PCBs with motor drivers are placed near to the fixed mirror. The part of the mechanism with the scanning mirrors which stands out of the FTIR case is covered by a safety shell. It increases instrument thermal stability and protects from Mars atmospheric dust. The mass of the pointing system with the electronics, locking system, and the safety shell is $0.8 \mathrm{~kg}$. The power consumption is $5 \mathrm{~W}$ (in Sun tracking regime).

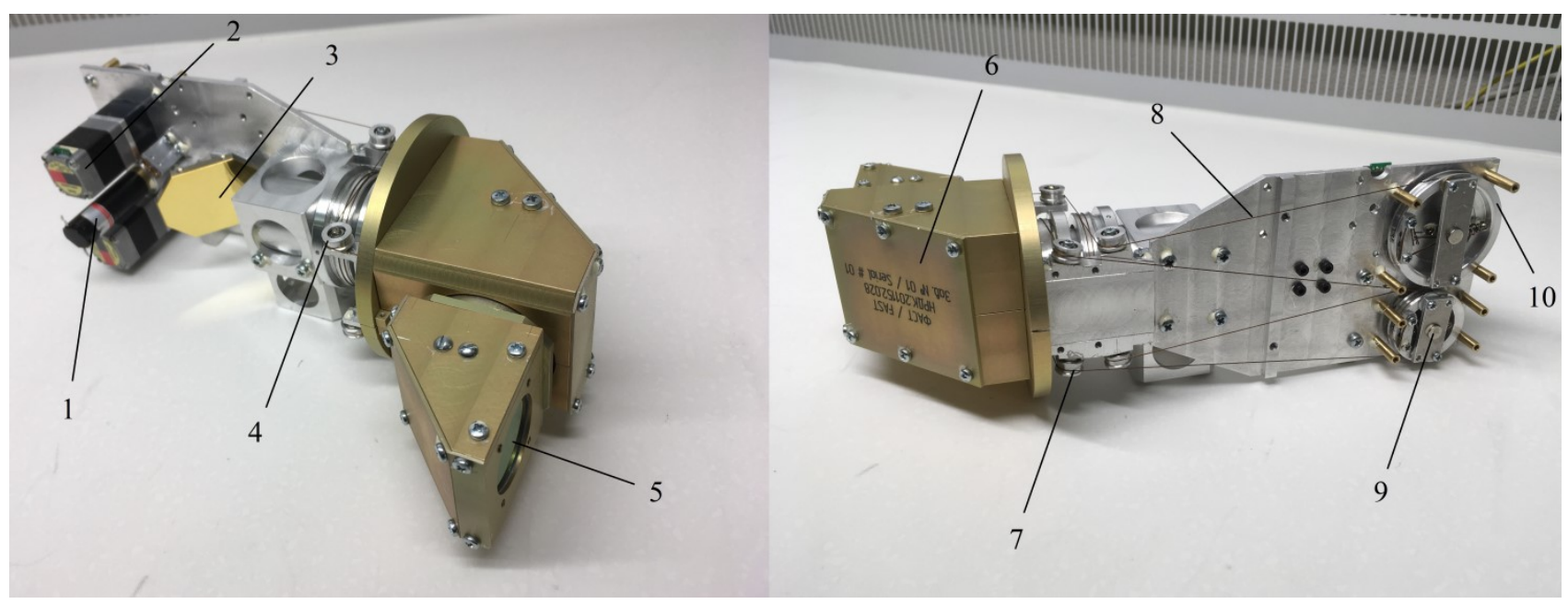

Figure 1. Two coordinates pointing system (different views). (1) locking mechanism; (2) geared stepper motor; (3) fixed flat mirror; (4) and (7) pulleys; (5) inlet window (Ge); (6) safety case; (8) steel rope; (9) magnet of the main pulley A; (10) main pulley B with encoder magnet. PCBs are not installed.

The optical scheme of the pointing system is described in [2] as part of the FTIR scheme. It consists of an inlet window (Ge) with an antireflection coating (AR) and a clear aperture of $28 \mathrm{~mm}$. Scanning and fixed mirrors are uniformly goldplated and have an aperture of $28 \mathrm{~mm}$. The thermopile array $(32 \times 32)$ used in sun tracking and tracing systems is part of the FTIR main detector telescope[2]. The array is involved into Sun tracking loop and is connected to the motor driver PCBs. It intercepts part of the light (about 25\%) passing through the spherical lens ( $\mathrm{ZnSe}$ ). The thermopile array has the same FOV (about $3^{\circ}$ ) as the main FTIR detector (pyroelectric).

A number of tests with different types of motors were performed before the final mechanical design was frozen. Application of geared (4403:1) brushed DC motors showed a pointing and Sun tracking accuracy of about one mrad. 
Five-phase geared stepper motors (1:50, 400 steps) showed a ten times better accuracy. The accuracy of a two-phase geared stepper motors (1:50, 200 steps) turned out to be the same (about $0.1 \mathrm{mrad}$ ) as for five-phase motors. However, the driving electronics for two-phase motors is more straightforward. Eventually, the two-phase geared stepper motors were applied.

The motor shafts in the pointing system prototype were connected to the main pulleys via couplings. Mechanical tests (vibration and shock) show that a locking system with such a connection of shaft and pulley is not required. The moment in the geared stepper motor, the friction between wires and pulleys and in the clutches is strong enough to hold the system when mechanical testing loads are applied. However, the size of the prototype was not compatible with the allocated FTIR outer dimensions. Optimization of the pointing system involved the removal of the couplings and implementing a simplified rigid shaft-pulley connection. This configuration could not withstand mechanical loads, and the scanner started slowly rotating during the vibration test. Thus, the locking system appears unavoidable to withstand mechanical stresses during take-off and landing. Such a system was developed and included in the pointing system design. It bases on a brushed DC motor and a slider. It locks the main pulley B (see Figure 1) during tests and launch, to unlock after landing on Mars.

The electronics of the pointing system is controlled by an ARM processor. It analyzes encoders and thermopile array data and operates geared stepper motors through a UART interface. The processor of the pointing system interprets telecommands from the FTIR central processor and sends back telemetry with the status of the pointer subsystem. In the sun searching and tracking regimes, an image from the thermopile array is analyzed by the operating processor. This image can also be transferred to the FTIR central processor upon respective telecommand.

\section{POINTING SYSTEM PERFORMANCE}

A simplified diagram of the pointing system scheme showing zero position, rotation limits, and directions is given in Fig. 2. The direction of the instrument IFOV can be calculated using the position of the two scanning mirrors via the encoder's readings and rope drive reduction coefficients. When the pointing system is at the initial position $(\alpha=0$ and $\beta=0)$, the encoders data equal to zero $\left(\alpha_{e}=0\right.$ and $\left.\beta_{e}=0\right)$, and the following equations determine the instrument's IFOV direction.

$$
\begin{gathered}
\beta=\beta_{e} \cdot K_{\beta}, \\
\alpha=\left(270^{\circ}+\beta_{e} \cdot K_{\beta}-\alpha_{e} \cdot K_{\alpha}\right),
\end{gathered}
$$

where $K_{\alpha}$ and $K_{\beta}$ are the reduction coefficients corresponding to main pulleys A and B: $K_{\alpha}=1, K_{\beta}=0.66$. These equations are used to program the IFOV pointing when the instrument operates in the thermal sounding regime (day or night time measurements) or during measurements of FTIR internal calibration source.

The sun searching mode starts with the initial direction indicated by a telecommand. The instrument's IFOV moves according to rectangular helix trajectory, with each following cycle shifted w.r.t. the previous one to the IFOV size $\left(\right.$ about $3^{\circ}$ ). The number of the scanning cycles is also set via telecommand. If the solar disk appears in the instrument IFOV the searching mode stops. The pointing system moves so that the sun image center remains at the specified pixel of the thermopile array (the pixel indexes $\mathrm{i}$ and $\mathrm{j}$ can be fractional). After that the sun tracking mode starts automatically. If no sun is found during the scanning, the pointing system stops and waits for the next telecommands. Auxiliary prestored parameters, such as the threshold or background can be updated by telecommands after analysis of the thermopile array images (if needed).

The Sun tracking operation algorithm is based on periodic analysis of the sun image and correction of the image position with respect to the specified one. The rate of the analysis and possible correction is $\leq 10 \mathrm{~Hz}$. This frequency is limited by the time constant of the thermopile array and the rate of the operating processor. The achieved accuracy (standard deviation) of pointing and tracking is $0.1 \mathrm{mrad}$ (Fig. 3). The accuracy is limited by mechanical properties of the pointing system, as the accuracy of the sun image position, calculated from the thermopile array data is about one order of magnitude higher. If the increment correction from the subsequent thermopile array image remains within $0.1 \mathrm{mrad}$ then no correction is applied (the system does not move), otherwise the IFOV correction is applied. 
The sun tracking accuracy influences the measurements of the FTIR, as moving the source within the IFOV distorts the interference picture in the detector plane. This effect can be considered as an additional source of noise. We estimated this influence quantitively by modelingt. The input data are the angular velocities of the instrument IFOV correction (9 $\left.\mathrm{mrad} \mathrm{s}^{-1}\right)$ and that of the sun at the Martian sky $\left(0.07 \mathrm{mrad} \mathrm{s}^{-1}\right)$ a. The relative motion of the sun within the instrument's IFOV was modeled with $10 \mathrm{~Hz}$ correction frequency for two different values of pointing accuracy $(1 \mathrm{mrad}$ and 0.1 $\mathrm{mrad}$ ). The correction performs a displacement of the IFOV instrument to a random point, where the standard deviation from the ideal pointing is given by estimated pointer accuracy. Each interferogram point for monochromatic light and specified difference in the interferometer shoulders is calculated as the total signal from the interference picture on the detector. It is uniquely defined by the angular size of the sun from the Martian orbit and its position whthin the instrument's IFOV. An integral over the solar spectrum (assumed as the Planck's curve for $6000 \mathrm{~K}$ ) for instrument spectral range $(1.7-17 \mu \mathrm{m})$ in the stationary interferometer configuration gives one interferogram point for the polychromatic light. The signal from the detector as a function of the optical path difference is an interferogram (set of the points) [10] which contains noise originating from the pointing inaccuracy. It was found that the effect of the pointing accuracy (for both $1 \mathrm{mrad}$ and $0.1 \mathrm{mrad}$ values) on the instrument's SNR is negligible comparing to the noise of the pyrodetector. The reason of the insignificance of the effect can be explained by a large separation between the frequency ranges for the sun signal $(20-100 \mathrm{~Hz})$ and the modulation conditioned by the finite accuracy of the tracking $(<10 \mathrm{~Hz})$.

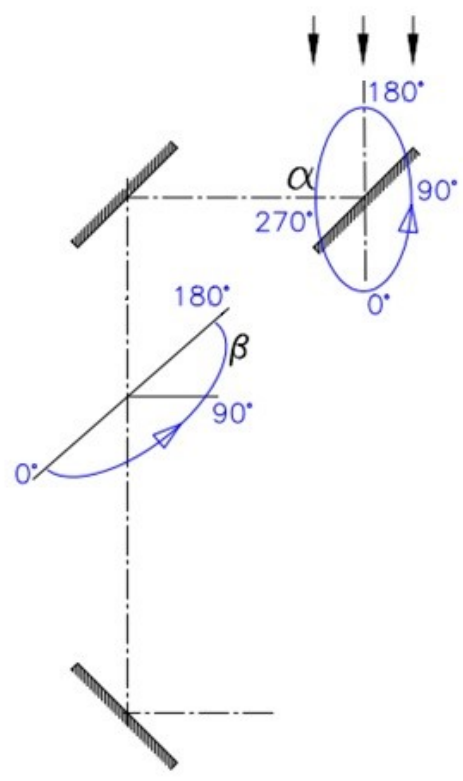

Figure 2. Simplified diagram of the two-coordinate pointing system showing the rotation limits $\left(0^{\circ}-360^{\circ}\right.$ for $\alpha$ and $0^{\circ}-180^{\circ}$ for $\beta$ ) and scanning directions. 


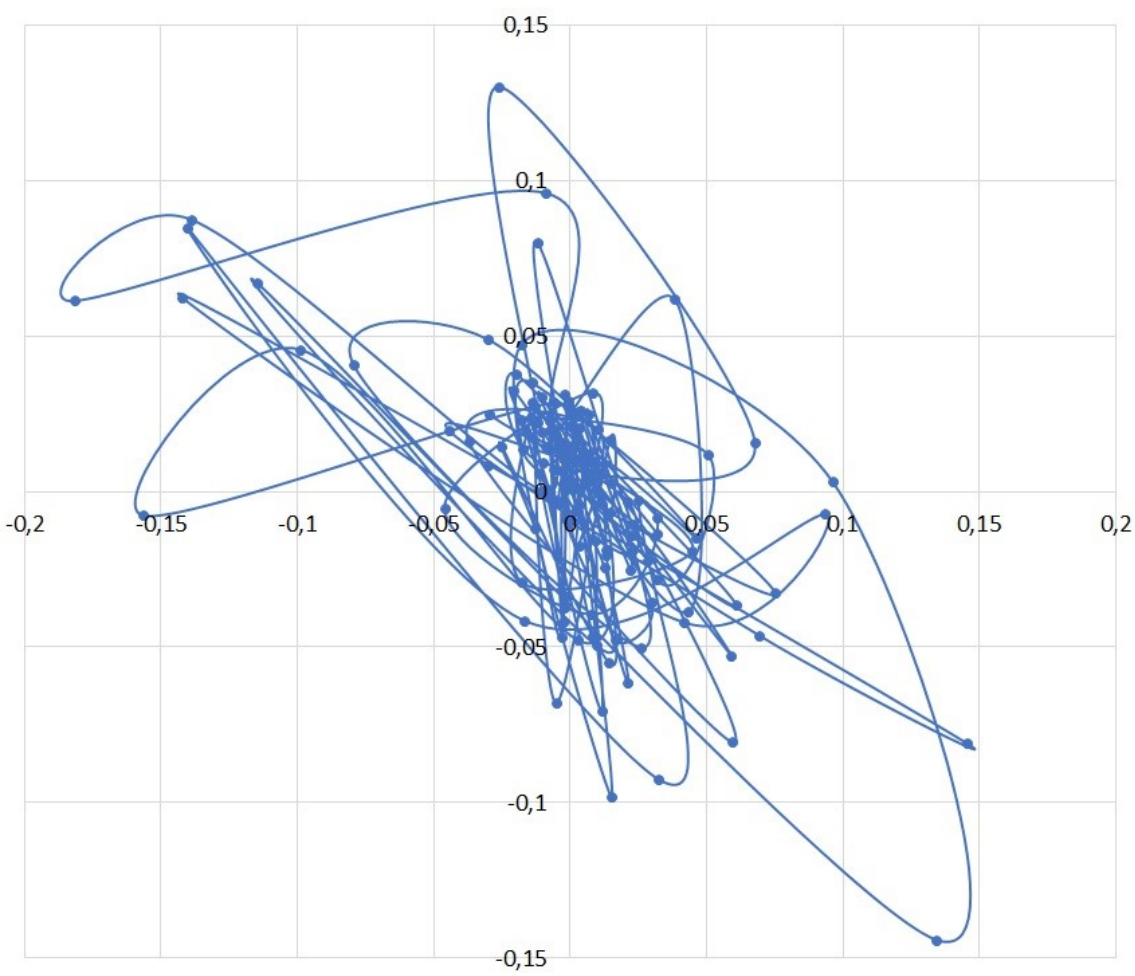

Figure 3. Diagram of the Sun tracking accuracy (in mrad) comparing with the specified value (for $15 \mathrm{~s}$ of operation).

\section{CONCLUSIONS}

The two-coordinate pointing system was developed as a part of FAST FTIR for ExoMars landing platform. Mechanically based on geared motors, pulleys and wire tensions, it allows the instrument's IFOV to be precisely pointed in any direction of the upper hemisphere, and to the body-mounted calibration blackbody. The system can find the sun on the sky, and to follow it in the sun-tracking regime. The accuracy of pointing and tracking is $0.1 \mathrm{mrad}(\sim 21$ arc seconds). The sun detector, a thermopile array serves in the tracking loop in the sun search and sun-tracking modes. A locking mechanism is used to withstand take-off and landing forces. The pointing system has a mass of $\sim 0.8 \mathrm{~kg}$ and occupies a volume of $250 \times 155 \times 85 \mathrm{~mm}^{3}$.

\section{ACKNOWLEDGEMENTS}

ExoMars is a space mission by ESA and Roscosmos. The development and fabrication of FAST was funded by Roscosmos. Alexey Shakun, Igor Stupin and Oleg Korablev acknowledge support from Russian Science Foundation (RSF grant 16-12-10453), which enabled to perform experiments and computer modeling described in sections 2 and 3.

\section{REFERENCES}

[1] Vaguine V.A., Egorov A.I., Zharkov A.V., Kotlov V.I., Moshkin B.E. "XY single-dish guidance system for spacecraft spectrometers," Physical Basis of Instrumentation, 5 (3), 92-96 (2016).

[2] A. Shakun, O. Korablev, B. Moshkin, A. Grigoriev, N. Ignatiev, I. Maslov, O. Sazonov, D. Patsaev, A. Kungurov, A. Santos-Skripko, A. Zharkov, I. Stupin, D. Merzlyakov, V. Makarov, F. Martinovich, Y. Nikolskiy, V. Shashkin 
"Fourier Transform Spectrometers for remote sensing of planetary atmospheres and surfaces," CEAS Space J., 9(4), 399-409 (2017).

[3] B.E. Moshkin, I.A. Maslov, O.V. Sazonov, I.G. Stupin "A Fourier-transform spectrometer for operation on Mars," Instrum Exp Tech, 62(2), 247-251 (2019).

[4] Rodionov, D., Korablev, O., Zelenyi, L.M., Vago, J., Bessonov, R., Lipatov, A., Shakun, A., Rodin, A.V., Vinogradov, I., Skulachev, D., Zakharov, A.V., Manukin, A., Mitrofanov, I.G., Skalsky, A., Gerasimov, M.V., Dehant, V., MartinTorres, J., "Mars atmospheric measurements planned at ExoMars 2020 Surface Platform," In: Forget, F. (ed) Mars Atmosphere: Modelling and http://wwwmars.lmd.jussieu.fr/granada2017/program_granada2017.htm (2017).

[5] O. Korablev, F. Montmessin, A. Trokhimovskiy, A.A. Fedorova, A.V. Shakun, A.V. Grigoriev, B.E. Moshkin, N.I. Ignatiev, et al., "The Atmospheric Chemistry Suite (ACS) of three spectrometers for the ExoMars 2016 Trace Gas Orbiter," Space Sci Rev, 214:7, doi: 10.1007/s11214-017-0437-6 (2018).

[6] Hanel, R., Conrath, B., Hovis, W., Kunde, V., Lowman, P., Maguire, W., Pearl, J., Pirraglia, J., Prabhakara, C., and Schlachman, B., "Investigation of the Martian environment by infrared spectroscopy on Mariner 9," Icarus 17, 423442 (1972).

[7] Grassi, D., Ignatiev, N. I., Zasova, L. V.; Maturilli, A., Formisano, V., Bianchini, G. A., Giuranna, M. "Methods for the analysis of data from the Planetary Fourier Spectrometer on the Mars Express Mission," Planet. Space. Sci., 53(10), 1017-1034 (2005).

[8] Smith, M. D., J. C. Pearl, B. J. Conrath and P. R. Christensen, "One Martian year of atmospheric observations by the thermal emission spectrometer," Geophysical Research Letters 28, 4263-4266 (2001).

[9] Smith, M. D., "Interannual variability in TES atmospheric observations of Mars during 1999-2003," Icarus 167, 148165 (2004).

[10]Bell, R.J., "Introductory Fourier transform spectroscopy", Academic Press, New York and London, (1972). 